Rev. salud pública. 13 (4): 560-571, 2011

\title{
Diabetes en México y Colombia: Análisis de la tendencia de años de vida perdidos, 1998-2007
}

\author{
Diabetes in Mexico and Colombia: analyzing potential lost years of \\ life, 1998-2007
}

\author{
Claudio A. Dávila-Cervantes ${ }^{1}$, Marcela Agudelo-Botero ${ }^{2}$ y
}

Laura E. Gloria-Hernández ${ }^{2}$

1 El Colegio de México. Distrito Federal, México. cadavila@colmex.mx

2 Instituto Nacional de Salud Pública. Cuernavaca, México. marcela.agudelo@insp.mx, laura.gloria@insp.mx

Recibido 28 Enero 2011/Enviado para Modificación 25 Julio 2011/Aceptado 15Agosto 2011

\section{RESUMEN}

Objetivo Analizar el nivel e impacto de la mortalidad por diabetes en México y Colombia entre 1998 y 2007 , por sexo y grupos de edad.

Material y métodos Se utilizaron estadísticas vitales de mortalidad por diabetes del Instituto Nacional de Estadística y Geografía (INEGI) en México y del Departamento Administrativo Nacional de Estadística (DANE) en Colombia. Se calcularon las tasas estandarizadas de mortalidad para ambos países, para hombres y mujeres. Después, se obtuvieron los años de vida perdidos (AVP) para cada uno de los años mencionados, a nivel nacional, por sexo y grupos quinquenales de edad (20-79 años).

Resultados En Colombia, la tasa estandarizada de mortalidad por diabetes pasó de 28,1 en 1998 a 29,3 muertes por cada 100000 personas en 2007 y en México se incrementó de 101,4 a 135,1. Tanto en México como en Colombia, las mujeres tuvieron tasas de mortalidad mayores que los hombres en todos los años. En cuanto a los AVP, en Colombia decrecieron 6,1 \% y en México subieron $30 \%$. Las mujeres colombianas y los hombres mexicanos fueron los que más años de vida perdieron por diabetes. Los grupos de edad más afectados fueron los de 60-69 años en Colombia y de 55-74 años en México.

Conclusiones En Colombia no se observa un incremento significativo de la mortalidad por diabetes en el periodo estudiado, mientras que en México el aumento en la mortalidad por esta causa es elevado, evidenciando los diversos contrastes entre países de América Latina.

Palabras Clave: Diabetes mellitus, mortalidad, tasa de mortalidad, Colombia, México (fuente: DeCS, BIREME). 


\section{ABSTRACT}

Objective Analysing the level and impact of mortality directly caused by diabetes by gender and age group in Mexico and Colombia between 1998 and 2007.

Material and Method Vital statistics provided by the Mexican Institute of Statistics and Geography (Instituto Nacional de Estadística y Geografía INEGI) and the Colombian Statistics Administrative Department (Departamento Administrativo Nacional de Estadística-DANE) were analysed regarding mortality caused by diabetes. Standardized mortality rates were calculated for both men and women; potential lost years of life (PLYL) were then obtained for each country, every year, by gender, using 5-year age-groups (20-79 years).

Results The standardized mortality rate for diabetes in Colombia rose from 28.1 deaths per 100000 people in 1998 to 29.3 in 2007; in Mexico it grew from 101.4 to 135.1 per 100000 people during the same period. Women had higher mortality rates than men for each year in both Mexico and Colombia. The PLYL decreased by $6.1 \%$ in Colombia and rose by $30 \%$ in Mexico. The 60-69 year-old age-group was most affected in Colombia compared to the 55-74 year-old bracket in Mexico.

Conclusions There was no significant increase in mortality from diabetes in Colombia during the study period compared to a high increase in mortality in Mexico, thereby contrasting two Latin-American countries' lifestyles.

Key Words: Diabetes mellitus, mortality, mortality rate, Colombia, Mexico (source: Mesh, NLM).

$\mathrm{L}$ a diabetes se perfila en la actualidad como uno de los grandes retos para la salud pública, tanto en países desarrollados como en países de ingresos medios y bajos (1). De acuerdo con la Organización Mundial de la Salud (OMS), la diabetes afecta entre un $10 \%$ y $15 \%$ de la población adulta de América Latina y el Caribe (2) y se estima que para 2025 la prevalencia alcance los 65 millones de personas (3). La diabetes no sólo es una enfermedad sino un síndrome heterogéneo en el que confluyen diversos padecimientos crónicos (afecta la retina, los riñones y el sistema nervioso, entre otros) (4) y se caracteriza por su elevada morbilidad y alto riesgo de muerte prematura (2).

A pesar de la importancia de la medición y seguimiento de esta patología, existen múltiples dificultades para elaborar una estimación precisa de la magnitud real de la enfermedad y su impacto en la población. En consecuencia, la comparación entre países se ve restringida, dado que la disponibilidad de información y metodologías de recolección de datos varían entre diversos contextos regionales y locales (5). Sin embargo, es fundamental establecer estudios que permitan observar el desarrollo de esta afección que se manifiesta indistintamente entre hombres y mujeres de todas las edades y de todas partes del mundo. 
Por lo general, los indicadores disponibles para evaluar las características de la mortalidad no develan las verdaderas consecuencias de una muerte temprana. Por lo tanto, el propósito central de este trabajo es analizar el nivel e impacto de la mortalidad por diabetes en México y Colombia entre 1998 y 2007, por sexo y grupos de edad. Ambos países se seleccionaron dado que tienen una prevalencia relativamente elevada de diabetes, en comparación con otros países de América Latina (5) y puesto que esta enfermedad se encuentra entre las principales causas de muerte de la población de cada país. Asimismo, México y Colombia transitan por etapas similares de sus procesos demográficos y epidemiológicos, lo que se refleja en el paulatino envejecimiento de su población, la cual está más expuesta al riesgo de padecer enfermedades crónicodegenerativas, entre las que destaca la diabetes (6).

\section{MATERIAL Y MÉTODOS}

Fuentes de información

La información de este estudio se obtuvo de las Estadísticas Vitales de Mortalidad del Instituto Nacional de Estadística y Geografía (INEGI) en México (7) y del Departamento Administrativo Nacional de Estadística (DANE) en Colombia (8). Los datos de defunciones se obtuvieron para los años 1998 a 2007, a nivel nacional, para ambos países, por sexo y grupos quinquenales de edad (20-79 años). Se consideraron para estos mismos grupos etarios, los fallecimientos por diabetes, según los criterios de la Clasificación Internacional de Enfermedades (CIE-10) (códigos E10 a E14).

Para el análisis, se tuvieron en cuenta las defunciones ocurridas al interior de cada país, quedando excluidos los siguientes casos: 1. Registros no especificados por edad y sexo; 2 . Muertes ocurridas en el exterior y; 3. Defunciones de menores de 20 años y mayores de 79. A partir de esto, se descartaron 18,3\% del total de registros de diabetes para México y 25,2 \% para Colombia entre 1998 y 2007.

\section{Métodos}

El análisis que se realizó es de tipo observacional y retrospectivo. Se calcularon tasas estandarizadas de mortalidad por diabetes, tanto para hombres como para mujeres. Para ello se usó como referente la población mundial total y por sexo para el periodo de estudio (1998-2007). La fórmula utilizada fue:

[1] Tasa estandarizada (diabetes) $^{=} \frac{\text { (Defunciones registradas por diabetes (año i) }}{\text { (Población mundial (año i) }} * 100000$ personas 
Posteriormente se procedió a calcular los AVP, con los cuales se trata de determinar cuántos años de vida en promedio pierde una población por muerte a determinadas edades; para ello se parte del supuesto de que la mortalidad debería haber sido nula entre dos edades elegidas. El sustento de esta técnica es que los que murieron deberían haber vivido hasta la edad superior del intervalo de edades dentro del que se analiza la mortalidad $(10,11)$. Su cálculo final es:

[2] ${ }_{\mathrm{u}} \mathrm{ap}_{\mathrm{j}, \mathrm{j}}=\sum_{x=a}^{v} \mathrm{u}, \mathrm{n}_{\mathrm{x}, \mathrm{j}}$

La suma de los AVP entre las edades $\alpha$ y $v$ da el promedio de AVP por la causa de muerte $j$ (diabetes) en el intervalo de edad de $u$ años (20-79).

El cálculo de los AVP parte de los resultados de tablas de mortalidad en lo relacionado con las defunciones y los sobrevivientes. De este modo se utilizaron tablas de mortalidad masculina y femenina para Colombia y México, previamente elaboradas por otros autores $(12,13)$. Los AVP se obtuvieron para cada uno de los años entre 1998 y 2007 a nivel nacional, por grupos de edad quinquenales (entre los 20 y 79 años) y por sexo.

\section{RESULTADOS}

Panorama de la mortalidad por diabetes en Colombia y México

Diabetes en Colombia

Para 2007, en Colombia, la diabetes ocupó la quinta causa de muerte a nivel nacional con un total de 7127 fallecimientos, de los cuales 99,3\% ocurrieron en personas de 20 años o más. De estos últimos, 56,8\% correspondieron a mujeres. En este mismo año, poco más de $50 \%$ de las defunciones por diabetes se concentraron en cuatro departamentos: Valle (15,2\%), Bogotá (15\%), Antioquia (14,7\%) y Santander (6,2\%). Entre 1998 y 2007, la tasa estandarizada total por diabetes sufrió un leve aumento, pasando de 28,1 a 29,3 por cada 100 mil personas; sin embargo, al desglosar la información por sexo, las mujeres tuvieron las tasas de mortalidad más elevadas con respecto a los hombres, patrón que se mantuvo a lo largo del periodo analizado (Figura 1).

Diabetes en México

En México, la diabetes fue la primera causa de muerte en 2007, con un total de 70512 defunciones, siendo 47,2\% entre hombres y $99,7 \%$ en personas de 20 años o más. En este mismo año, seis entidades federativas agruparon 
$50,7 \%$ de las muertas por esta causa: Estado de México (13,8 \%), Distrito Federal, (12,1\%), Veracruz (7,5\%), Jalisco (6,3\%), Puebla $(5,6 \%)$ y Guanajuato $(5,4 \%)$. La mortalidad por diabetes en el territorio mexicano ha mostrado un incremento elevado en su tasa estandarizada global, pasando de 101,4 en 1998 a 135,1 por cada 100 mil habitantes en 2007, representando esto un aumento de $33,3 \%$. Las mujeres tienen tasas de mortalidad que superan el promedio nacional y la tasa de los hombres, situación que se asemeja a la de Colombia. No obstante, mientras que las mujeres han aumentado su tasa en $26,6 \%$, los hombres lo han hecho en $41,8 \%$ entre 1998 y 2007 (Figura 2).

Figura 1. Tasas estandarizadas de mortalidad* por diabetes en población de 20 años y más, por sexo. Colombia, 1998-2007

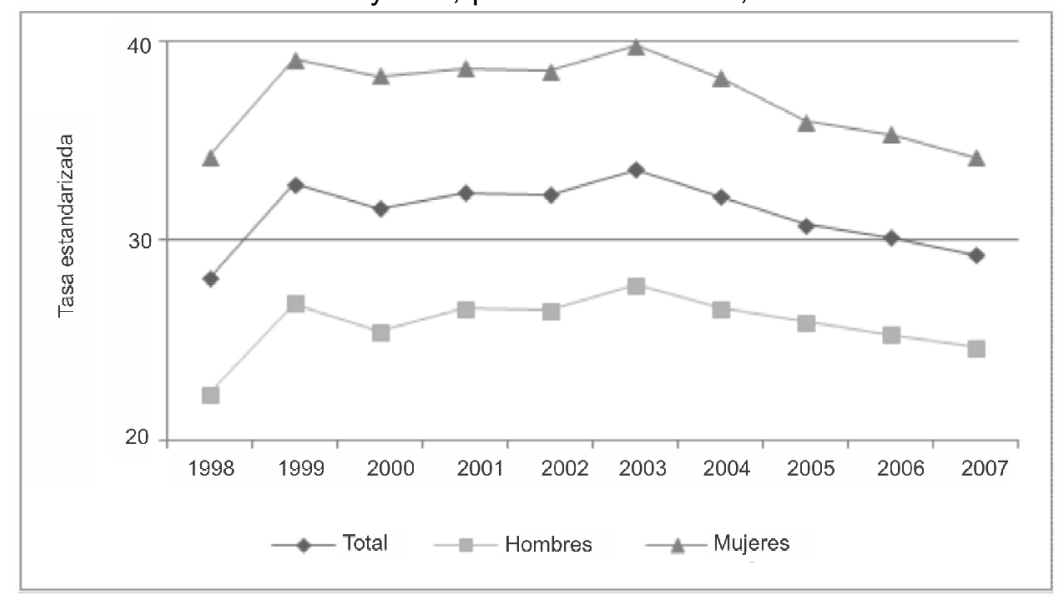

* Estandarizada con la población mundial (total y por sexo), 1998-2007

Fuente: Departamento Administrativo Nacional de Estadística (DANE), Estimaciones 1985-2005 y proyecciones de población 2006-2020 por sexo y edad; DANE, Estadísticas Vitales de Mortalidad en Colombia 1998-2006. División de Población del Departamento de Asuntos Económicos y Sociales de la Secretaría de las Naciones Unidas. World Population Prospects: The 2010 Revision. Elaboración propia.

Análisis de Años de Vida Perdidos

En Colombia, el promedio de los AVP por diabetes en la población de 20-79 años de edad en 1998 era mayor que en 2007, sufriendo una disminución de 6,1\%, en cambio, en México, el valor de este indicador tuvo un incremento de $30 \%$. Esto significa que para Colombia pasó de 0,35 a 0,33 y en México de 0,85 a 1,1.

Tendencia por sexo

En 1998, los AVP en Colombia por diabetes en mujeres de 20-79 años de edad eran superiores a los AVP de los hombres. Esta situación se mantuvo a lo largo 
de todo el periodo de estudio. El indicador de los hombres subió de 0,27 a 0,28 y en las mujeres se mantuvo en promedio en 0,34. En 2003 se observó la mayor pérdida de años de vida a consecuencia de la diabetes en ambos sexos (Figura 3).

Figura 2. Tasas estandarizadas de mortalidad* por diabetes en población de 20 años y más, por sexo. México, 1998-2007

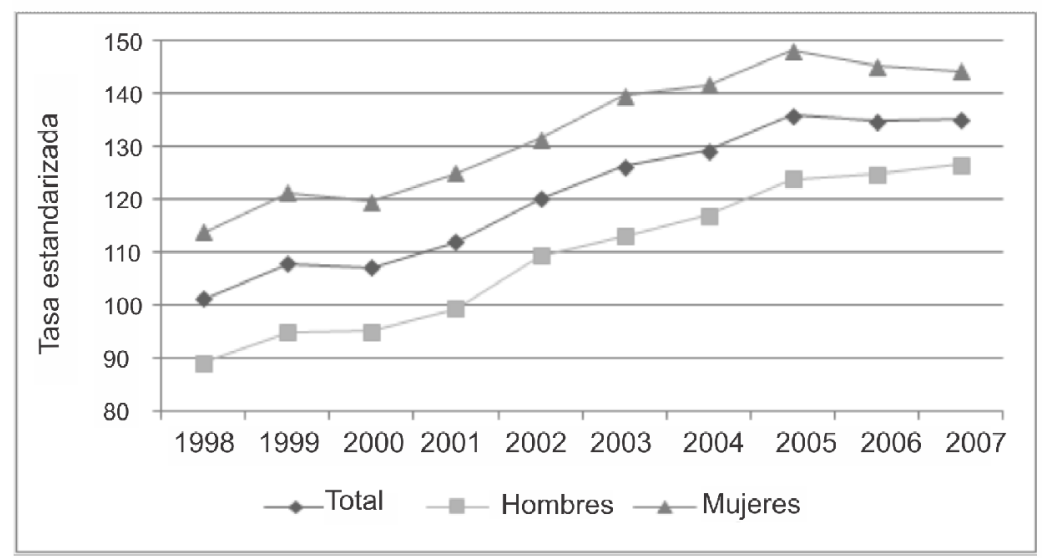

* Estandarizada con la población mundial (total y por sexo), 1998-2007.

Fuente: Instituto Nacional de Estadística y Geografía (INEGI), Estadísticas Vitales de Mortalidad en México 1998-2007; Consejo Nacional de Población (CONAPO), Proyecciones de Población, 1970-2030. División de Población del Departamento de Asuntos Económicos y Sociales de la Secretaría de las Naciones Unidas. World Population Prospects: The 2010 Revision. Elaboración propia.

Figura 3. Tendencia en el promedio de años de vida perdidos* por diabetes en México y Colombia según sexo, 1998-2007

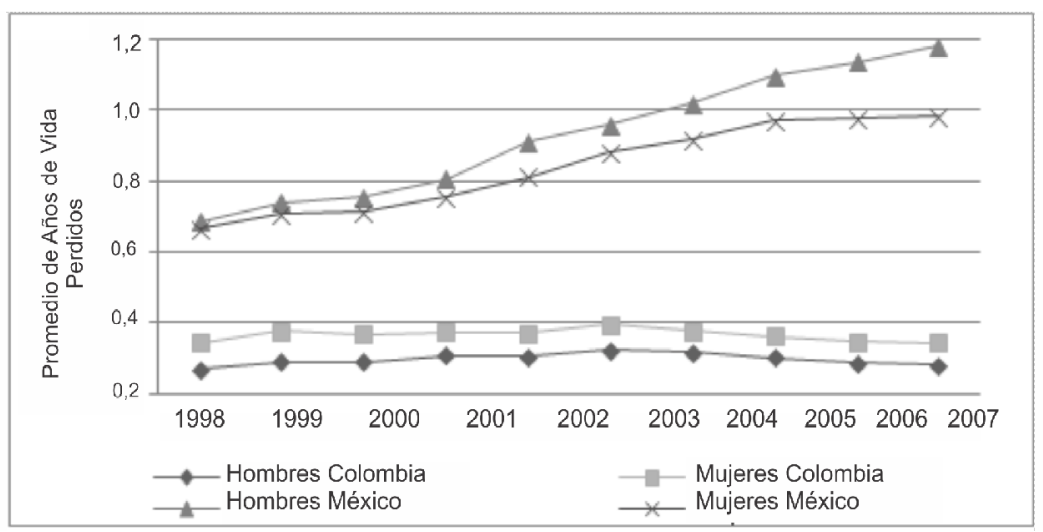

* En población entre los 20 y 79 años.

Fuente: Elaboración propia a partir de información del Departamento Administrativo Nacional de Estadística (DANE), Colombia, 1998-2007 y del Instituto Nacional de Estadística y Geografía (INEGI), México 1998-2007. 
En México, los AVP por diabetes fueron superiores para hombres en todos los años de análisis. Para los hombres el indicador pasó de 0,7 a 1,2, lo que significó un aumento de 71,8 \% entre 1998 y 2007; en las mujeres pasó de 0,7 en 1998 a 1 en 2007, lo que equivale a un incremento de poco más de $48 \%$. Dado el patrón de comportamiento del indicador, el año en el cual se perdieron más años de vida fue 2007 (Figura 3).

Años de vida perdidos por grupos de edad

Los hombres colombianos que perdieron más años de vida por diabetes durante el periodo de estudio se encontraban entre los 55-69 años. El grupo de edad que presentó el incremento más alto en el promedio de AVP fue el de 70-79 años, en tanto que el de 55-59 años fue el que mostró la mayor disminución.

Tabla 1. Promedio de años de vida perdidos por diabetes según grupos de edad y sexo. Colombia, 1998-2007

\begin{tabular}{ccccccccccc}
\hline & \multicolumn{7}{c}{ Hombres } \\
Edades & 1998 & 1999 & 2000 & 2001 & 2002 & 2003 & 2004 & 2005 & 2006 & 2007 \\
\hline $20-24$ & 0.002 & 0.003 & 0.003 & 0.002 & 0.002 & 0.003 & 0.003 & 0.002 & 0.003 & 0.002 \\
$25-29$ & 0.005 & 0.003 & 0.003 & 0.003 & 0.002 & 0.003 & 0.003 & 0.003 & 0.002 & 0.004 \\
$30-34$ & 0.003 & 0.005 & 0.006 & 0.005 & 0.004 & 0.004 & 0.004 & 0.005 & 0.003 & 0.003 \\
$35-39$ & 0.005 & 0.007 & 0.007 & 0.009 & 0.008 & 0.005 & 0.007 & 0.006 & 0.006 & 0.006 \\
\hline $40-44$ & 0.007 & 0.013 & 0.012 & 0.011 & 0.010 & 0.008 & 0.011 & 0.008 & 0.006 & 0.005 \\
$45-49$ & 0.017 & 0.018 & 0.018 & 0.016 & 0.016 & 0.015 & 0.016 & 0.016 & 0.014 & 0.015 \\
$50-54$ & 0.030 & 0.033 & 0.032 & 0.035 & 0.038 & 0.036 & 0.033 & 0.039 & 0.030 & 0.032 \\
$55-59$ & 0.044 & 0.039 & 0.048 & 0.051 & 0.043 & 0.051 & 0.051 & 0.042 & 0.046 & 0.040 \\
$60-64$ & 0.048 & 0.057 & 0.053 & 0.056 & 0.055 & 0.062 & 0.064 & 0.057 & 0.049 & 0.051 \\
\hline $65-69$ & 0.057 & 0.059 & 0.056 & 0.056 & 0.061 & 0.065 & 0.061 & 0.059 & 0.061 & 0.060 \\
$70-74$ & 0.035 & 0.038 & 0.040 & 0.047 & 0.049 & 0.051 & 0.045 & 0.047 & 0.048 & 0.043 \\
$75-79$ & 0.013 & 0.017 & 0.015 & 0.017 & 0.017 & 0.020 & 0.018 & 0.019 & 0.020 & 0.021 \\
Total & 0.267 & 0.291 & 0.291 & 0.307 & 0.305 & 0.322 & 0.315 & 0.303 & 0.288 & 0.281 \\
\hline & & & & & Mujeres & & & & & \\
$20-24$ & 0.005 & 0.003 & 0.003 & 0.003 & 0.002 & 0.002 & 0.003 & 0.001 & 0.003 & 0.002 \\
$25-29$ & 0.004 & 0.004 & 0.003 & 0.005 & 0.003 & 0.004 & 0.003 & 0.003 & 0.003 & 0.002 \\
$30-34$ & 0.004 & 0.004 & 0.003 & 0.003 & 0.004 & 0.004 & 0.003 & 0.002 & 0.003 & 0.004 \\
\hline $35-39$ & 0.006 & 0.006 & 0.006 & 0.007 & 0.005 & 0.005 & 0.005 & 0.004 & 0.003 & 0.005 \\
$40-44$ & 0.010 & 0.013 & 0.010 & 0.009 & 0.011 & 0.011 & 0.008 & 0.010 & 0.008 & 0.009 \\
$45-49$ & 0.027 & 0.021 & 0.019 & 0.022 & 0.023 & 0.023 & 0.022 & 0.019 & 0.019 & 0.023 \\
$50-54$ & 0.032 & 0.038 & 0.035 & 0.040 & 0.034 & 0.045 & 0.040 & 0.041 & 0.040 & 0.034 \\
$55-59$ & 0.046 & 0.055 & 0.052 & 0.050 & 0.048 & 0.052 & 0.055 & 0.051 & 0.048 & 0.051 \\
$60-64$ & 0.066 & 0.072 & 0.065 & 0.069 & 0.067 & 0.071 & 0.065 & 0.062 & 0.059 & 0.049 \\
$65-69$ & 0.066 & 0.076 & 0.078 & 0.071 & 0.079 & 0.078 & 0.074 & 0.073 & 0.070 & 0.071 \\
$70-74$ & 0.056 & 0.060 & 0.068 & 0.069 & 0.072 & 0.076 & 0.070 & 0.066 & 0.065 & 0.065 \\
$75-79$ & 0.024 & 0.026 & 0.025 & 0.027 & 0.026 & 0.027 & 0.030 & 0.029 & 0.030 & 0.029 \\
Total & 0.345 & 0.378 & 0.367 & 0.375 & 0.372 & 0.396 & 0.378 & 0.362 & 0.348 & 0.344 \\
\hline
\end{tabular}

Fuente: Elaboración propia a partir de información del Departamento Administrativo Nacional de Estadística (DANE), Colombia, 1998-2007 
En lo que respecta a las mujeres colombianas, el mayor número de AVP estuvo entre los 60-74 años. Las que elevaron el promedio de AVP correspondieron al grupo de edad 70-79 años, mientras que las de 60-64 años fueron las que más redujeron el valor de este indicador (Tabla 1).

En 1998, el nivel más elevado de AVP entre los varones mexicanos se dio en el grupo de edad 60-69 años, a diferencia de 2007 donde se concentró en el rango de edad comprendido entre los 55-74 años. Es importante resaltar que en todos los grupos etarios se evidenció una tendencia ascendente en el promedio de AVP. Los hombres que sufrieron la mayor pérdida de años por diabetes entre 1998 y 2007 fueron los de 55-64 y los de 70-74 años. Al inicio del periodo, las mujeres mexicanas que perdieron más AVP estaban en los grupos etarios 65-69 y 70-74 años, y para 2007 se concentraban entre los 60-74 años. Las mujeres presentaron incrementos en cuanto al promedio de los AVP entre 1998 y 2007, siendo mayor entre los 60-74 años (Tabla 2).

Tabla 2. Promedio de años de vida perdidos por diabetes según grupos de edad y sexo. México, 1998-2007

\begin{tabular}{ccccccccccc}
\hline & \multicolumn{10}{c}{ Hombres } \\
Edades & 1998 & 1999 & 2000 & 2001 & 2002 & 2003 & 2004 & 2005 & 2006 & 2007 \\
\hline $20-24$ & 0.001 & 0.001 & 0.001 & 0.001 & 0.001 & 0.001 & 0.001 & 0.001 & 0.001 & 0.001 \\
$25-29$ & 0.002 & 0.002 & 0.002 & 0.002 & 0.002 & 0.002 & 0.002 & 0.002 & 0.002 & 0.002 \\
$30-34$ & 0.004 & 0.004 & 0.004 & 0.005 & 0.004 & 0.005 & 0.005 & 0.006 & 0.005 & 0.006 \\
$35-39$ & 0.007 & 0.007 & 0.007 & 0.007 & 0.007 & 0.008 & 0.008 & 0.009 & 0.009 & 0.010 \\
$40-44$ & 0.017 & 0.016 & 0.017 & 0.017 & 0.020 & 0.021 & 0.023 & 0.024 & 0.024 & 0.024 \\
$45-49$ & 0.034 & 0.035 & 0.038 & 0.040 & 0.047 & 0.048 & 0.050 & 0.056 & 0.058 & 0.061 \\
$50-54$ & 0.069 & 0.075 & 0.075 & 0.086 & 0.095 & 0.099 & 0.107 & 0.117 & 0.122 & 0.127 \\
$55-59$ & 0.111 & 0.118 & 0.121 & 0.124 & 0.143 & 0.155 & 0.166 & 0.180 & 0.182 & 0.199 \\
$60-64$ & 0.134 & 0.148 & 0.150 & 0.157 & 0.182 & 0.184 & 0.198 & 0.211 & 0.217 & 0.230 \\
$65-69$ & 0.141 & 0.150 & 0.150 & 0.162 & 0.186 & 0.195 & 0.199 & 0.211 & 0.221 & 0.224 \\
$70-74$ & 0.117 & 0.131 & 0.135 & 0.143 & 0.156 & 0.175 & 0.184 & 0.199 & 0.208 & 0.209 \\
\hline $75-79$ & 0.050 & 0.053 & 0.056 & 0.060 & 0.065 & 0.066 & 0.072 & 0.079 & 0.084 & 0.085 \\
Total & 0.686 & 0.739 & 0.754 & 0.804 & 0.909 & 0.958 & 1.016 & 1.094 & 1.133 & 1.178 \\
& & & & & Mujeres & & & & & \\
$20-24$ & 0.002 & 0.002 & 0.002 & 0.003 & 0.002 & 0.002 & 0.002 & 0.003 & 0.002 & 0.002 \\
$25-29$ & 0.003 & 0.003 & 0.003 & 0.003 & 0.003 & 0.003 & 0.004 & 0.004 & 0.004 & 0.004 \\
$30-34$ & 0.004 & 0.004 & 0.004 & 0.005 & 0.005 & 0.005 & 0.006 & 0.006 & 0.005 & 0.006 \\
$35-39$ & 0.006 & 0.008 & 0.007 & 0.008 & 0.008 & 0.008 & 0.009 & 0.008 & 0.009 & 0.009 \\
$40-44$ & 0.016 & 0.016 & 0.016 & 0.017 & 0.018 & 0.020 & 0.020 & 0.022 & 0.021 & 0.022 \\
$45-49$ & 0.029 & 0.030 & 0.033 & 0.033 & 0.037 & 0.039 & 0.043 & 0.044 & 0.044 & 0.043 \\
$50-54$ & 0.057 & 0.062 & 0.062 & 0.065 & 0.070 & 0.076 & 0.079 & 0.082 & 0.086 & 0.085 \\
$55-59$ & 0.085 & 0.090 & 0.088 & 0.094 & 0.098 & 0.108 & 0.113 & 0.122 & 0.118 & 0.123 \\
$60-64$ & 0.117 & 0.122 & 0.123 & 0.133 & 0.140 & 0.154 & 0.156 & 0.166 & 0.171 & 0.168 \\
$65-69$ & 0.143 & 0.148 & 0.149 & 0.151 & 0.165 & 0.183 & 0.188 & 0.196 & 0.193 & 0.192 \\
$70-74$ & 0.138 & 0.150 & 0.150 & 0.161 & 0.177 & 0.191 & 0.201 & 0.211 & 0.216 & 0.218 \\
\hline $75-79$ & 0.065 & 0.071 & 0.073 & 0.080 & 0.087 & 0.092 & 0.094 & 0.108 & 0.108 & 0.111 \\
Total & 0.664 & 0.707 & 0.711 & 0.753 & 0.810 & 0.880 & 0.914 & 0.970 & 0.975 & 0.983 \\
\hline
\end{tabular}

Fuente: Elaboración propia a partir de información del Instituto Nacional de Estadística y Geografía (INEGI), México 1998-2007 


\section{DISCUSIÓN}

La diabetes es una enfermedad de alta prioridad en países en desarrollo, dada la magnitud de su impacto sobre la calidad de vida de la población y los elevados gastos para su atención y tratamiento, así como otros costos indirectos debido a la pérdida de productividad por invalidez y mortalidad prematura $(1,3,14)$. Con datos de 2000 se calculó que el costo anual por esta enfermedad en América Latina fue de US\$ 65216 millones al año (15) y se estimó que el número de personas viviendo con diabetes en esta región ascendió a 45 millones en 2010 (2).

Aunque la región muestra contrastes diversos en la incidencia, prevalencia y mortalidad por esta enfermedad, se ha enfatizado en la necesidad de establecer políticas integrales de atención para atenuar y controlar los efectos adversos que se derivan de su presencia. Algunos estudios señalan que determinadas complicaciones relacionadas con la diabetes pueden ser prevenibles; del mismo modo, la información y educación permanente a la población podría redundar en estilos de vida saludables que reduzcan los factores de riesgo y por ende la morbilidad y mortalidad asociada con este padecimiento $(1,5)$. Sin embargo, estas medidas tendrán que ser replanteadas en los contextos específicos y según los requerimientos de cada país.

México y Colombia son un ejemplo que permite ilustrar dimensiones heterogéneas del fenómeno dentro del ámbito latinoamericano. En Colombia, la prevalencia estimada de diabetes en personas adultas se situó en $8,2 \%$ en 2000 y en México fue de 14,9\%, con lo cual se clasifica estos dos países con una prevalencia media-alta y alta (5). Estas cifras pueden diferir considerablemente, teniendo en cuenta que en ambos países, al igual que en América Latina en general, no existen registros continuos de vigilancia epidemiológica que permitan hacer mediciones más precisas.

En este estudio se comprobó que la mortalidad por diabetes en adultos ha descendido en Colombia, mientras que en México se ha incrementando en los últimos años. En Colombia, esta tendencia requiere ser analizada con más detalle, ya que esto podría estar explicándose porque las personas están falleciendo por otras causas, incluso antes de llegar a edades en las que puedan desarrollar esta enfermedad. En este último país las principales causas de muerte están asociadas con las agresiones (homicidios) y accidentes de motor, afectando en mayor medida a la población en edad productiva. Por su parte, en México, a pesar de los importantes esfuerzos de manejo clínico del 
paciente diabético, se ha referido un inadecuado control metabólico de estas personas por lo que aún no se ha logrado declinar el nivel de la mortalidad (16).

El promedio de AVP en Colombia disminuyó 6,1 \% entre 1998 y 2007 y en México se incrementó $30 \%$. Tal como se ha visto en otras investigaciones $(3,17)$ y se constató en este estudio, la tasa estandarizada de mortalidad por diabetes fue más alta entre las mujeres que en los varones y de nueva cuenta más alta para México que para Colombia. Pese a ello, se resalta que en México los hombres sufrieron más AVP que las mujeres, en contraste con Colombia donde se observó una situación inversa. Esto se debe a la edad en la que murieron las personas, siendo más temprana entre los hombres mexicanos y las mujeres colombianas.

Estos hallazgos son de gran relevancia puesto que conocer las características sociodemográficas de las personas que mueren por diabetes sirve para trazar rutas concretas de acción sobre grupos vulnerables. De ahí la urgencia de mantener registros vitales oportunos, actualizados y consistentes. La OMS advierte que existen serias dificultades en los reportes de mortalidad por diabetes, debidas principalmente a que las personas que padecen la afección fallecen por otro tipo de complicaciones como las cardiovasculares y la nefropatía, lo que esconde la verdadera magnitud del problema (2). Esto afecta también los cálculos derivados de dichas estadísticas, como por ejemplo en la estimación de los AVP.

Por otro lado, como consecuencia del incremento en la esperanza de vida y la disminución de la fecundidad, se espera tener una mayor población envejecida en riesgo de padecer diabetes (18). El grupo etario de 65 años o más en Colombia pasará de 6,3\% del total de su población en 2007 a 7,5\% en 2015; en México representó 5,5\% en 2007 y subirá a 6,8\% en 2015. Aunado a esto, la Organización Panamericana de la Salud (OPS) advierte que la obesidad es un detonante que contribuye al aumento de casos de diabetes. En Colombia, el Instituto Colombiano de Bienestar Familiar (ICBF) reportó que en $2005,46 \%$ de la población de 18 a 64 años sufría de exceso de peso (sobrepeso u obesidad), siendo mayor en las mujeres $(49,6 \%)$ que entre los varones (39,6 \%) (19). Al igual, en México, en la Encuesta Nacional de Salud y Nutrición de 2006 (ENSANUT), se refirió que cerca de $70 \%$ de las personas entre los 30 y los 60 años tenían problemas de sobrepeso y obesidad (71,9\% mujeres, 66,7 \% hombres) (20). Partiendo de este único factor de riesgo se podría afirmar que 5 de cada 10 colombianos y 7 de cada 10 mexicanos adultos tendrían altas probabilidades de padecer diabetes. 
Este trabajo constituye un acercamiento inicial al impacto de la mortalidad por diabetes en dos países con rasgos diferenciados, por lo que se sugiere continuar con análisis que incorporen otras variables económicas, geográficas y demográficas de los individuos. En la medida que esto sea posible, se dispondrá de más evidencias que ayuden a dirigir las decisiones en el tema, especialmente en lo que tiene que ver con tareas de información, prevención de la enfermedad, acceso y calidad de los servicios de salud; al igual que en el direccionamiento adecuado de los recursos humanos, técnicos y financieros.

Finalmente, hay que subrayar que la estimación de los AVP se ve restringida al tener que delimitarse un rango de edad, en este caso en particular entre los 20 y 79 años, lo que provoca que se subestime el impacto total de la mortalidad por diabetes, el cual puede ser más alto que el establecido en este trabajo. Sin embargo, la mayor parte de individuos, cuya muerte se debió a esta causa, quedó incluida en esta investigación

\section{REFERENCIAS}

1. Barquera S. Prevención de la diabetes: Un problema mundial. Salud Pública Méx. 2003; 45(5):413-414

2. OMS. Iniciativa de Diabetes para las Américas (DIA): Plan de Acción para América Latina y El Caribe 2001-2006 [OPS/OMS]. División de prevención y control de enfermedades/ Programa de enfermedades no-transmisibles/Organización Panamericana de la Salud/Organización Mundial de la Salud. Washington DC; 2001.

3. King H, Aubert R, Herman W. Global Burden of Diabetes, 1995-2025. Prevalence, numerical estimates, and projections. Diabetes Care. 1998; 21(9): 1414-1431.

4. Cabrera E, Suárez L, Díaz O. Nuevos criterios para clasificar la diabetes. Rev Cubana Endocrinol. 2000; 11(1):51-55.

5. OPS. La diabetes en las Américas, Boletín Epidemiológico [OPS]. OPS. 2001; 22(2):1-3.

6. OMS. Informe sobre la salud en el mundo 2008: La atención primaria de salud, más necesaria que nunca. Ginebra. OMS; 2001.

7. INEGI. Estadísticas de Mortalidad. [Internet]. Disponible en: http://www.inegi.org.mx/est/ contenidos/espanol/proyectos/continuas/vitales/bd/mortalidad/ MortalidadGeneral.asp?s=est\&c=11144. Consultado Agosto de 2010.

8. DANE. Estadísticas Vitales de Mortalidad en Colombia 1982-2007. [Base de datos en CDROM]. Bogotá; 2009.

9. United Nations Department of Economic and Social Affairs (UNDESA). World Population Prospects: The 2008 Revision [Internet]. Disponible en: http://esa.un.org/unpd/wpp/ index.htm. Consultado Septiembre de 2010.

10. Arriaga E. Comentarios sobre algunos índices para medir el nivel y el cambio de la mortalidad. Estudios Demográficos y Urbanos 1996; 11:5-30.

11. Arriaga E. Los años de vida perdidos: Su utilización para medir el nivel y cambio de la mortalidad. Notas de Población 1996; 24:7-38.

12. DANE. Series de Población 1985-2020. Indicadores demográficos y tablas abreviadas de mortalidad nacionales y departamentales 1985-2005 Internet]. Disponible en: http://www.dane.gov.co/ index.php?option=com_content\&task=category\&sectionid=16\&id=496\&Itemid=996. Consultado Julio de 2010. 
13. Mina A. Funciones de sobrevivencia empleadas en el análisis demográfico. Papeles de Población. 2001; 28:131-154.

14. Majikela B, Isiavwe A, Levitt N. Monitorización de la diabetes en países en desarrollo. Diabetes Voice. 2006; 51 (Num. especial): 28-31.

15. Barceló A, Aedo C, Rajpathak S, Roble S. The cost of diabetes in Latin America and the Caribbean. Bulletin of the World Health Organization. 2003; 81(1):19-28.

16. Hernández $\mathrm{C}$, Elnecavé $\mathrm{A}$, Huerta $\mathrm{N}$, Reynoso N. Análisis de una encuesta poblacional para determinar los factores asociados al control de la diabetes mellitus en México. Salud Pública Méx. 2011; 53(1):34-39.

17. Moreno L. Epidemiología y diabetes. Rev Fac Med. Universidad Nacional Autónoma de México. 2003; 44(1):35-37.

18. Aschner P. Epidemiología de la diabetes en Colombia. Av. Diabetol. 2010; 26(2):95-100.

19. Ojeda G, Ordoñez M, Ochoa LH. Encuesta nacional de la situación nutricional en Colombia, 2005. Instituto Colombiano de Bienestar Familiar. Bogotá; 2006.

20. Olaiz G, Rivera J, Shamah T, Rojas R, Villalpando S, Hernández M, Sepúlveda J. Encuesta Nacional de Salud y Nutrición 2006. Instituto Nacional de Salud Pública. México; 2006. 